\title{
Reduced-Complexity Nonlinear Inverse Synthesis Transmission Method with Polarization Division Multiplexing
}

\author{
Stella Civelli ${ }^{(1,2)}$, Sergei K. Turitsyn ${ }^{(2)}$, Marco Secondini ${ }^{(1)}$, Jaroslaw E. Prilepsky ${ }^{(2)}$ \\ (1) TeCIP Institute, Scuola Superiore Sant'Anna, Pisa, Italy, stella.civelli@santannapisa.it \\ (2) AIPT, Aston University, Birmingham, United Kingdom
}

Abstract Using numerical simulations, we demonstrate the feasibility of polarisation division multiplexing nonlinear inverse synthesis transmission. A reduced-complexity processing for dual polarization nonlinear Fourier-based transmission schemes is proposed and studied.

\section{Introduction}

Optical transmission methods based on the nonlinear Fourier transform (NFT) for signal (de)modulation have recently attracted a great deal of attention ${ }^{1}$ due to their robustness to fiber nonlinear effects, which are believed to limit the performance of current-generation transmission systems. The NFT exploits the integrability of the master models for fiber channels-either the scalar nonlinear Schrödinger equation (NLSE) for single-polarization signals or the vector Manakov equation (ME) for dual-polarization signals ${ }^{2}$ - to represent the optical signal through a nonlinear spectrum that evolves in a linear manner along the channel ${ }^{3,4}$. The nonlinear inverse synthesis (NIS) ${ }^{5}$ is an NFT-based transmission scheme that encodes the information into the nonlinear spectrum, such that deterministic propagation effects can be exactly reversed at the receiver (RX). Recently, polarization division multiplexing (PDM) NFT-based schemes ${ }^{6,7}$ have been studied as a tool to increase the line throughput. In this work we consider PDM nonlinear inverse synthesis (PDM-NIS) as a dual polarization extension of NIS. The standard PDM-NIS assumes that we use the NFT associated with the ME for encoding and decoding information, in agreement with the channel model. To reduce the method's complexity, we also propose here the PDM-NIS NLS $_{\text {ap- }}$ proach that exploits the scalar NFT (associated with the NLSE) to independently process each polarization component of the optical signal, in place of the more involved vector NFT associated with the ME.

\section{Numerical methods for the NFT for ME}

In this section, we briefly describes two methods for the numerical computation of the NFT and the inverse NFT (INFT) for the $\mathrm{ME}^{4}$ in the case of fiber links with anomalous dispersion.

For the computation of the nonlinear spectrum from the time-domain signal (direct NFT), we propose a generalization of the well-studied BoffettaOsborne method ${ }^{8}$, within which we compute the scattering data (the NFT spectrum) $a(\lambda), b_{1}(\lambda)$, and $b_{2}(\lambda)$, which depends on the nonlinear frequency $\lambda$, assuming a piece-wise constant approximation of the dual-polarization time domain signal $\mathbf{q}(t)=\left(q_{1}(t), q_{2}(t)\right)$. The dual-component continuous nonlinear spectrum is obtained as

$$
\boldsymbol{\rho}(\lambda)=\left(\rho_{1}(\lambda), \rho_{2}(\lambda)\right)=\left(b_{1}(\lambda), b_{2}(\lambda)\right) / a(\lambda) .
$$

Let $\mathbf{q}(t)=0$ outside $[-T, T]$, and consider the uniform grid $t_{n}=-T+(n-1) \delta$ for $n=1, \ldots, N+$ 1 , with discretization step $\delta=2 T / N$. Let $\mathbf{q}^{(n)}=$ $\mathbf{q}\left(t_{n}\right)$ and $U^{(n)}$ be the transfer matrix

$$
U^{(n)}=\left(\begin{array}{ccc}
c_{0}-j \lambda s_{0} & q_{1}^{(n)} s_{0} & q_{2}^{(n)} s_{0} \\
-q_{1}^{(n) *} s_{1} & c_{1}+j \lambda s_{1} & 0 \\
-q_{2}^{(n) *} s_{2} & 0 & c_{2}+j \lambda s_{2}
\end{array}\right),
$$

where $c_{k}=\cosh \left(\delta d_{k}\right)$ and $s_{k}=\sinh \left(\delta d_{k}\right) / d_{k}$ for $k=0,1,2, d_{0}=\left(-\lambda^{2}-\left|q_{1}^{(n)}\right|^{2}-\left|q_{2}^{(n)}\right|^{2}\right)^{1 / 2}$, and $d_{k}=\left(-\lambda^{2}-\left|q_{k}^{(n)}\right|^{2}\right)^{1 / 2}$ for $k=1,2$. Compute $\phi=$ $\left(\phi_{1}, \phi_{2}, \phi_{3}\right)^{\mathrm{T}}=U^{(N+1)} \ldots U^{(1)}[1,0,0]^{\mathrm{T}}$ and obtain $a(\lambda)=\phi_{1} e^{j \lambda(2 T+\delta)}, b_{1}(\lambda)=\phi_{2}$, and $b_{2}(\lambda)=\phi_{3}$.

Regarding the INFT, the time domain signal $\mathbf{q}(t)$ can be obtained from the nonlinear spectrum by generalizing the Nystromconjugate-gradient method used to solve the Gelfand-Levitan-Marchenko equation (GLME) in the scalar NLSE case $^{9}$. The GLME for the $\mathrm{ME}^{4}$ is a two-dimensional integral equation in which the nonlinear spectrum appears through the dual-component kernel function $\mathbf{F}(y)=$ $\left(F_{1}(y), F_{2}(y)\right)^{4}$, defined (in the case of $a(\lambda)$ having only simple zeros) as

$$
\mathbf{F}(x)=\frac{1}{2 \pi} \int_{-\infty}^{+\infty} \boldsymbol{\rho}(\lambda) e^{j \lambda x} d \lambda-j \sum_{i=1}^{N} \mathbf{C}_{i} e^{j \lambda_{i} x},
$$



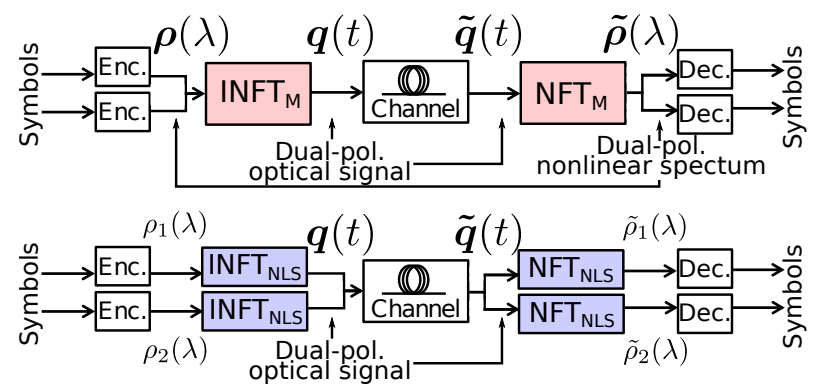

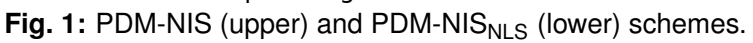

where $\mathbf{C}_{i}$ is the discrete spectrum component corresponding to the eigenvalue $\lambda_{i}$, for any $i=$ $1, \ldots, N$ such that $\lambda_{i} \in \mathbb{C}^{+}$is a simple zeros of $a(\lambda)$. Let $t$ be the time instant in which the solution has to be found, and consider the uniform grid $\alpha_{m}=(m-1) 2 \delta$ for $m=1, \ldots, M$ where $M$ is chosen such that $\|\mathbf{F}(y)\|=0$ for $y \geq 2 t+\alpha_{M}$. Let $D$ be the diagonal matrix characterizing the composite Simpson's quadrature rule, i.e., with diagonal $2 \delta / 3\{1,4,2,4, \ldots, 4,2,4,1\} ; H_{k}$ be the matrices $\left(H_{k}\right)_{m, n}=F_{k}\left(2 t+\alpha_{m}+\alpha_{n}\right)$ for $m, n=1, \ldots, M$ and $k=1,2$, and $\mathbf{f}_{k}$ the vector $\left(\mathbf{f}_{k}\right)_{m}=F_{k}\left(2 t+\alpha_{m}\right)$ for $m=1, \ldots, L$ and $k=1,2$. The GLME is reduced to the system

$$
\left\{\begin{array}{c}
A_{1,1} \mathbf{b}_{1}+A_{1,2} \mathbf{b}_{2}+D \mathbf{b}_{1}=D \mathbf{f}_{1}^{*} \\
A_{2,1} \mathbf{b}_{1}+A_{2,2} \mathbf{b}_{2}+D \mathbf{b}_{2}=D \mathbf{f}_{2}^{*}
\end{array},\right.
$$

with unknown $L$-dimensional column vectors $\mathbf{b}_{1}$ and $\mathbf{b}_{2}$, where $A_{m, n}=D H_{m}^{\dagger} D H_{n} D$ and $H_{m^{-}}$ $s$ are Hankel matrices. The discrete system (1) can now be solved with the conjugate gradient method, using FFTs for fast products between the matrices $H_{m}$ and respective vectors. Finally, the time domain solution (in the fixed time instant $t$ ) is found from the first component of the unknown vectors as $q_{k}(t)=-2\left(\mathbf{b}_{k}\right)_{1}$.

\section{Simulation setup and results}

In this work, we consider two PDM transmission schemes based on the NFT: PDM-NIS and PDM-NIS ${ }_{\text {NLS }}$, shown schematically in Fig. 1 in the upper and lower parts, respectively. Both schemes are considered in the same scenario as in $^{10}$, rearranged for the dual polarization case. At the transmitter (TX), $N_{s}$ information symbols $\left(N_{s} / 2\right.$ per polarization) with quadrature phase-shift keying modulation are independently mapped onto two nonlinear spectra $\rho_{1}(\lambda)$ and $\rho_{2}(\lambda)^{10}$. The optical signal is obtained as follows: PDM-NIS uses one joint INFT $\mathbf{M}$ to obtain $\mathbf{q}(t)$ from $\boldsymbol{\rho}(\lambda)=\left(\rho_{1}(\lambda), \rho_{2}(\lambda)\right)$, while PDM-NIS NLS $_{\text {per- }}$ forms two independent INFT NLS $_{\text {so obtain the two }}$ components $q_{k}(t)$ from the corresponding $\rho_{k}(\lambda)$ for $k=1,2$. At the $\mathrm{RX}$, the TX operations are in- verted: PDM-NIS performs a joint $\mathrm{NFT}_{\mathrm{M}}$ to obtain the dual polarization nonlinear spectrum, while PDM-NIS ${ }_{N L S}$ uses two independent NFT NLS $_{\text {of }}$ each signal's polarization to get the two nonlinear spectrum components. Then, deterministic propagation effects are removed multiplying for $e^{4 j \lambda^{2} \mathcal{L}}$, with $\mathcal{L}$ being the normalized channel length ${ }^{7}$, and matched filtering and sampling are used to recover the transmitted information.

In an ideal noiseless scenario, the PDM-NIS is exact since $T X$ and $R X$ employ $\mathrm{NFT}_{M}$ in agreement with the signal propagation (governed by the

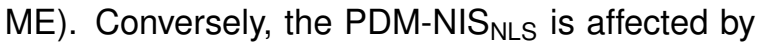
ISI due to the mismatch between signal propagation (governed by the ME and, hence, including an interaction between the two polarizations) and the encoding/decoding techniques (which neglect such interaction). The impact of this mismatch is addressed later in this section. However,

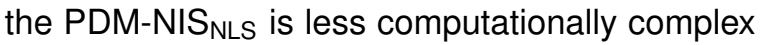
(i.e., it requires less floating-point operations).

In our simulations the link, of length $L=$ $2000 \mathrm{~km}$, is a standard single mode fiber with an ideal distributed amplification, with: group velocity dispersion parameter $\beta_{2}=-20.39 \mathrm{ps}^{2} / \mathrm{km}$, nonlinear coefficient $\gamma=1.22 \mathrm{~W}^{-1} \mathrm{~km}^{-1}$, attenuation $\alpha_{\mathrm{dB}}=0.2 \mathrm{~dB} / \mathrm{km}$, and spontaneous emission noise was simulated in a standard way ${ }^{10,11}$. The symbol rate is $R_{s}=50 \mathrm{GBd}$ per polarization. We inserted $N_{z}=800$ guard symbols per polarization between bursts to avoid their interaction during propagation ${ }^{10}$. The NFTs are computed as described in the previous section, with oversampling factor of 4 samples per symbol (i.e., $\delta=1 /\left(4 R_{s}\right)$ ): this value is enough to eliminate the impact of numerical inaccuracies on our results.

System performance, evaluated through simulations and presented in terms of $Q^{2}$-factor ${ }^{10}$, is shown in Fig. 2(a) as a function of the average power $P_{s}=E_{s} R_{s}$, where $E_{s}$ is the average energy per information symbol. Figure 2(a) qualifies the performance obtained for different $N_{s}$ (marked with the same color) for the PDM-NIS system with solid lines, and for PDM-NIS ${ }_{\text {NLS }}$ with dashed ones. The comparison between

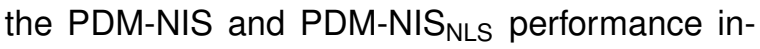
dicates that (i) for larger $N_{s}$, i.e., when the optimal performance is higher, PDM-NIS ${ }_{\mathrm{NLS}}$ performs worse than PDM-NIS, as the expected consequence of the mismatch between the channel and the processing technique; (ii) for smaller $N_{s}$, i.e, when the optimal performance is lower, PDM-NIS ${ }_{\text {NLS }}$ performs better than PDM-NIS, con- 

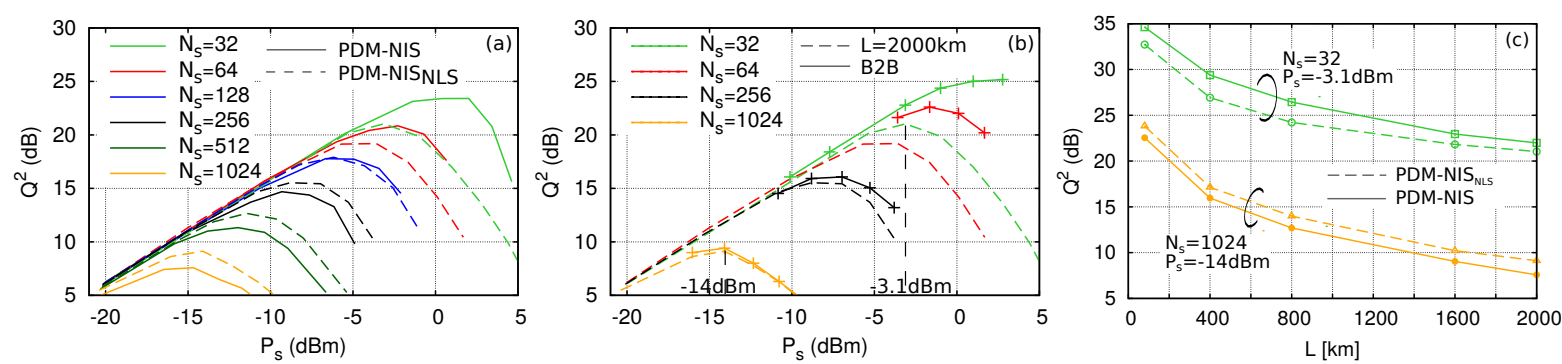

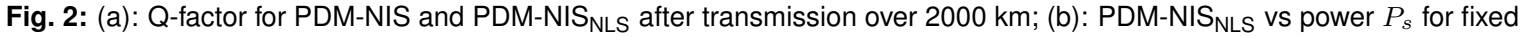
noise power; (c): PDM-NIS and PDM-NIS ${ }_{\text {NLS }}$ vs propagation distance $L$ for fixed powers.

trary to the previous case. Such a counterintuitive behavior can be explained by noting that, while PDM-NIS ${ }_{\text {NLS }}$ processes each polarization component of the received noisy signal by an independent $\mathrm{NFT}_{\mathrm{NLS}}$, PDM-NIS uses a single $\mathrm{NFT}_{\mathrm{M}}$ to jointly process the two components, in fact processing a doubled-energy noisy signal. We conjecture that this fact increases the impact of noise on the nonlinear spectrum (which, in the scalar case, is known to depend on the energy of the signal ${ }^{10,11}$ ). Overall, Fig. 2(a) shows that when the achievable performance is higher, the optimal performance is obtained by the PDM-NIS scheme, while when the achievable performance is worse, it is better to use the PDM-NIS ${ }_{\text {NLS }}$ one, both in terms of complexity and performance.

The impact of the mismatch in PDM-NIS ${ }_{N L S}$ is shown in Fig. 2(b), which compares the performance with the actual fiber and in back-toback (B2B) configuration (but with same total accumulated noise), as a function of $P_{s}$. For smaller $N_{s}$, the B2B performance (when there is no propagation and, hence, no mismatch) is significantly higher than after $2000 \mathrm{~km}$ (when the mismatch becomes relevant). Conversely, for larger $N_{s}$, the noise impact on the system is stronger than the mismatch (recall that the impact of noise on the nonlinear spectrum increases with $N_{s}$, as demonstrated in the singlepolarization case ${ }^{10,11}$ ), such that the latter becomes negligible and the same performance is achieved in B2B and after $2000 \mathrm{~km}$. In this case, PDM-NIS $_{\text {NLS }}$ performs better than PDM-NIS, as shown in Fig. 2(a). Finally, Fig.2(c), which com-

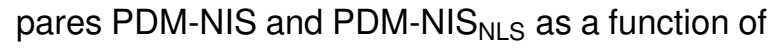
the propagation length for a fixed launch power

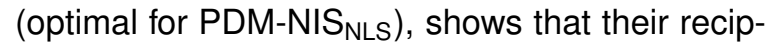
rocal behavior remains unchanged for different $L$.

\section{Conclusions}

In this work, we considered two PDM NFTbased schemes: PDM-NIS uses the ME integrability, while PDM-NIS ${ }_{\text {NLS }}$ uses a simplified scalar $\mathrm{NFT}_{\text {NLS }}$ processing and has lower complexity. A comparison between the two schemes reveals a peculiar behaviour that depends on the relative impact of noise and of the deterministic mismatch

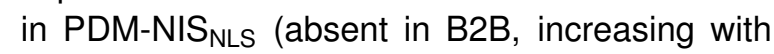
propagation length): when the former is domi-

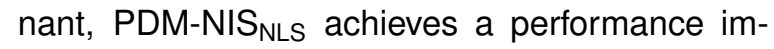
provement of about $1 \mathrm{~dB}$ compared to PDM-NIS, while PDM-NIS performs better in the other case.

\section{Acknowledgements}

This work was supported in part by the Erasmus + mobility programme grant and the Leverhulme Project RPG-2018-O63."

\section{References}

[1] S. K. Turitsyn, et. al.. Nonlinear Fourier transform for optical data processing and transmission: advances and perspectives. Optica, 4(3):307-322, 2017.

[2] C. R. Menyuk and B. S. Marks. Interaction of polarization mode dispersion and nonlinearity in optical fiber transmission systems. J. Lightw. Technol., 24(7):2806, 2006.

[3] V. E. Zakharov and A. B. Shabat. Exact theory of two-dimensional self-focusing and one-dimensional self-modulation of waves in nonlinear media. Soviet Physics-JETP, 34: 62-69, 1972.

[4] S. V. Manakov. On the theory of two-dimensional stationary self-focusing of electromagnetic waves. Soviet Physics-JETP, 38(2):248-253, 1974.

[5] S. T. Le, J. E. Prilepsky, and S. K. Turitsyn. Nonlinear inverse synthesis for high spectral efficiency transmission in optical fibers. Opt. Express, 22(22):26720-26741, 2014.

[6] S. Gaiarin, et. al.. Dual-polarization nonlinear fourier transform-based optical communication system. Optica, 5(3):263-270, 2018.

[7] J.-W. Goossens, et. al.. Polarization-division multiplexing based on the nonlinear fourier transform. Optics express, 25(22):26437-26452, 2017.

[8] G. Boffetta and A. R. Osborne. Computation of the direct scattering transform for the nonlinear Schroedinger equation. J. Comput. Phys., 102(2):252-264, 1992.

[9] A. Aricò, G. Rodriguez, and S. Seatzu. Numerical solution of the nonlinear Schrödinger equation, starting from the scattering data. Calcolo, 48(1):75-88, 2011.

[10] S. Civelli, E. Forestieri, and M. Secondini. Why noise and dispersion may seriously hamper nonlinear frequencydivision multiplexing. IEEE Photon. Technol. Lett., 29(16):1332-1335, 2017.

[11] S. A. Derevyanko, J. E. Prilepsky, and S. K. Turitsyn. Capacity estimates for optical transmission based on the nonlinear Fourier transform. Nat. Commun., 7:12710, 2016. 